\title{
The Development of the Method for the Calculation of the Shaping Force in the Production of Vehicle Wheel Rims
}

\author{
Ruslan Puzyr $^{1 *}$, Volodymyr Kukhar ${ }^{2}$, Alexander Maslov ${ }^{1}$, Yevhenii Shchipkovskyi ${ }^{3}$ \\ ${ }^{I}$ Kremenchuk Mykhailo Ostrohradskyi National University \\ ${ }^{2}$ Pryazovskyi State Technical University \\ ${ }^{3}$ Scientific production company «Tehvagonmash», Ukraine, \\ *Corresponding author E-mail: puzyruslan@gmail.com
}

\begin{abstract}
The objective of the paper is the development of the method for the calculation of the required force of shaping of a horizontal shapeforming machine used in the preproduction workflow. The design of a machine for horizontal shape-forming of wheel steel rims enables quick readjusting and transfer to another rim shape, which is significant under the conditions of frequent alteration of the product nomenclature. We developed the calculation method based on the assumptions of the deformations continuity, the constancy of the volume at plastic deformation and axisymmetric stressed state as well as the local application of the load. The research resulted in obtaining the analytical dependences for the calculation of the force of shaping of a wheel rim, taking into account the degree of deformation, the mechanical characteristics of the metal, hardening as well as the design and technological factors of the process. The topicality of the development consists in the necessity for the high-quality and the least expensive choice of the equipment of the required rated power when designing a new workflow. The experimental data, carried out both under the production and laboratory conditions, confirmed the adequacy of the theoretical formulas. It is determined by the assumptions and solutions obtained based on the mathematical apparatus of the technical theory of the shells of revolution. The clearness of the method will make it possible to algorithmize the calculation process quite easily and to use it as a subprogram in an applied complex of technical preproduction. It will also allow determining the shaping process most significant factors influencing the value of the force without the direct use of the calculation. We recommend to use it at enterprises producing vehicle steel wheels by the method of radial-rotation shaping.
\end{abstract}

Keywords: closed shell; deformation hardening; force of shaping; plastic deformation; wheel rim.

\section{Introduction}

Under the modern pace of development of science and technology the basic requirement to a highly productive and highly efficient production consists in a break-even termination of the manufacture of the developed product. It also includes the transfer to the output of any-size production lot of new items which may differ from each other. The main role here consists in the increase of the operational efficiency in the field of technical preproduction, i. e. reduction of the design and technological preparation, wide use of the unified and typical technological equipment with the increase of its service life [1].

\subsection{Analysis of Recent Studies and Publications}

Carrying out various types of design and technological calculations at the present stage of wheel production development, engineers pay the greatest attention to the results obtained based on the computational modeling with the visualization by application program package $[2,3]$. However, these calculations are rather labor-intensive and sometimes give contradictory results, as the level of training, the work experience and the notions of different researchers and engineers as to the regularities of the shaping processes are also different. We know several methods for the calculation of the power parameters of shapeforming machines manufacturing wheel rims from technical literature. Methods of different authors $[4,5]$ fundamentally differ in the choice of the initial conditions and adopted assumptions. The latter includes the neglect of the value of the elastic deformation or taking into account the alteration of the workpiece thickness in the deformation zone, etc. The results of calculations by these methods are different, the discrepancy is especially big, up to 1.5-2 times and more in the calculations of the shapeforming machine torques. Moreover, one should bear in mind that the calculation of the energy-power parameters of the radialrotation shaping processes can be only approximate. It is impossible to exactly determine the initial conditions (the variation of the workpiece geometric dimensions and the original material mechanical characteristics) in this case. Due to it, one cannot consider useful the determination of the elastic deformation of every roller in the methods of some authors [6]. It results in the necessity for additional laborious calculations.

\subsection{Statement of the Objective and Tasks of the Study}

The objective of this research consists in the development of a method for the calculation of the force at the radial-rotation method of wheel rims production and the verification of its adequacy to the experimental data.

To achieve the stated objective we posed and solved the following problems: 
- to theoretically determine the deformations of the wheel rim workpiece in the tangential direction based on the kinematic coupling of the shaping rollers in the shape-forming process;

- to account the metal hardening at deformation by the linear approximation of the hardening curve;

- to obtain the analytical dependences for the calculation of the shaping force with the use of the mathematical apparatus of the technical theory of the shells of revolution;

- to compare the calculation results with experimental measurement of the shaping force.

\section{The Basic Part of the Study}

As a number of researches $[7,8]$ indicate, the theoretical analysis of the process of radial-rotation shaping represents a rather complicated problem. It is due to the locality of the load application to the shell of revolution as well as to the simultaneous compression and stretch of different sections of the workpiece in the tangential direction. That is why the authors of paper [7] use a numerical approach and the researchers in paper [8] obtain the solutions based on harmonic functions. To determine the deformations in the tangential direction we propose the analysis of the movement of the shaping roller axes and determining the deformations by the movement. It essentially simplifies the solutions obtained based on the relations of the deformations and stresses according to the deformation theory of plasticity.

According to the required deformation in one step (determined by the design dimensions of the rim) shells are assigned by the value of feed in one turn $s_{i}=s_{i b}+s_{i g}$, with the workpiece radius $R_{O}$ (determined by the design dimensions of the area of the groove and the area of the seat) and deforming rollers radius $R_{i n}, R_{o u}$ (assigned by the design department based on the shaping of wheel rims of similar dimension types). The average value of the relative deformation in the tangential direction of the i-th turn of the shell can be determined by the following dependence (Fig. 1):

- the reduction zone

$\varepsilon_{i t}=\frac{A_{0 g}-\left(A_{i g}\right)}{A_{O g}}=\frac{\left(R_{o u}-R_{0}\right)-\left(R_{o u}-R_{O}-s_{i g}\right)}{R_{o u}-R_{O}}=\frac{s_{i g}}{R_{o u}-R_{O}} ;$

- the expansion zone

$\varepsilon_{i t}=\frac{A_{i b}-A_{o b}}{A_{0 b}}=\frac{\left(R_{0}+R_{i n}\right)-\left(R_{0}+R_{i n}+s_{i b}\right)}{R_{0}+R_{i n}}=\frac{s_{i b}}{R_{0}+R_{i n}}$,

where $\mathrm{A}_{0 \mathrm{~g}}, \mathrm{~A}_{0 \mathrm{~b}}$ - the initial distances between the roller and workpiece axes in the areas of the groove and the seat, respectively.

We calculate the value of feed in the areas of the seat and the groove, respectively, Fig. 1

$$
\begin{aligned}
& s_{i b}=s_{i}\left(1-0,57 \frac{\left(R_{0}-R_{i n}\right)^{2}}{\left(R_{o u}+R_{i n}\right)^{2}}\right), \\
& s_{i g}=0,57 \frac{\left(R_{0}-R_{\text {in }}\right)^{2}}{\left(R_{\text {ou }}+R_{\text {in }}\right)^{2}} s_{i},
\end{aligned}
$$

where $\mathrm{R}_{0}, \mathrm{R}_{\mathrm{ou}}, \mathrm{R}_{\mathrm{in}}$ - the radii of the workpiece, the external and the internal rollers, respectively.

Then we pass from the feed to the final dimensions of the groove $-h_{f}=h_{g}=h_{1}+h_{2}$, where $h_{1}$ and $h_{2}-$ the depth of the seat and the groove, respectively, and $\sum s_{i b}=h_{1}, \sum s_{i g}=h_{2}$.

We determine the angle of the grip of the workpiece by the roller in the expansion zone $\left(\varphi_{0 \mathrm{~b}}\right)$ and the reduction zone $\left(\varphi_{0 \mathrm{~g}}\right)$ $\varphi_{0 b}=\arccos \left(1-\frac{s_{i b}}{R_{i n}}\right)$,

$\varphi_{0 g}=\arccos \left(1-\frac{s_{i g}}{R_{o u}}\right)$.

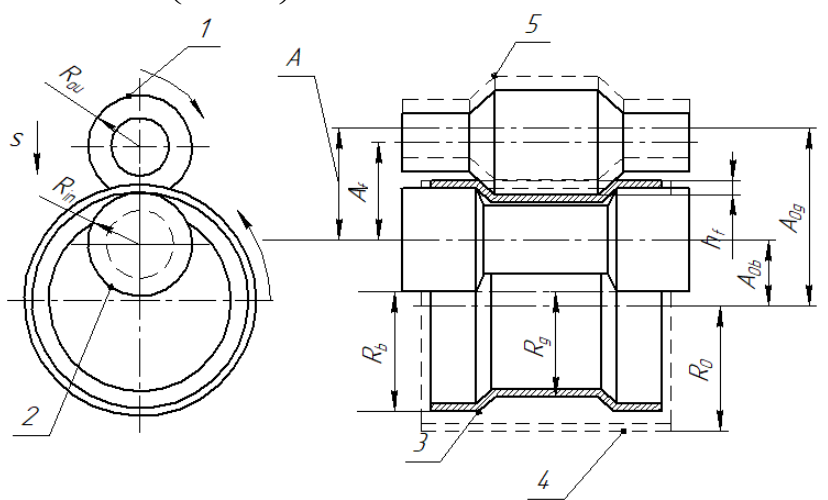

Fig. 1. The deformation of the semifinished product by the forming rollers: 1 - the external roller, 2 - the internal roller, 3 - the semifinished product, 4 - the workpiece, 5 - the initial position of the upper roller; $h_{f}-$ the groove depth, $\mathrm{A}_{0 \mathrm{~g}}$ - the initial distance between the centers of the workpiece and the external roller, $\mathrm{A}_{0 \mathrm{~b}}$ - the initial distance between the centers of the workpiece and the internal roller, $A_{f}-$ the final distance between the centers of the rollers, A - the initial distance between the centers of the rollers.

As a wheel rim workpiece represents a shell of revolution, we performed the analysis of the workpiece deformation with the use of the mathematical apparatus of the technical theory of shells. At the first step it is shaped - cylindrical without preliminary upsetting and conic after the upsetting (expansion); at the second and further steps it is stepwise combining different revolution surfaces (a half-torus, a truncated cone, a cylinder). The wheel rim is represented as a thin shell. The boundary between the thin and thick shells is conditional and usually determined by relation $(h / R)_{\text {max }} \approx 1 / 20$, where $h$ - the shell thickness, $R$ - the radius of the median surface curvature.

Parameter $(h / R)$ of most shells is much less than $1 / 20$. In the theory of thin shells all the members of the order of $(h / R)$ are neglected in comparison with one as the use of Kirchhoff's hypotheses, which are the basis for the technical theory of shells, gives the same error.

The theory of the calculation of thin shells bases on the following Kirchhoff-Love's hypotheses [9]:

1. The hypothesis of normals invariability. It is assumed that the normals to the median surface of the shell are not distorted and remain perpendicular to the deformed median surface. This hypothesis relates the deformed state in an arbitrary point of the shell wall and the alteration of the geometry of its median surface. It enables the reduction of the research of the shell deformation to the research of the deformation of its median surface.

2. The hypothesis of non-pressure of one shell layer against another. According to this hypothesis, the normal stresses in the areas parallel to the median surface are assumed to be equal to zero, i.e. the stressed state is considered as a flat one and not 3D.

These hypotheses reduce the problem to the research of the deformation of the shell median surface. Besides, only the shells whose sag is small in comparison with the thickness are considered.

However, in this instance it is rather difficult to obtain a trailing analytical solution in the case of local stressing too. That is why we performed further simplification of the balance equations by means of V.Z. Vlasov's semi-zero-moment theory [10-12].

The wish to simplify the general theory of cylindrical shells and the small value in some case of moments $M_{1}, H_{1}$ and $H_{2}$, and, consequently, the small value of $Q_{1}$ encouraged V.Z. Vlasov to create the so-called semi-zero-moment theory of cylindrical shells 
This theory, apart from the general hypotheses of KirchhoffLove's shell theory, contains additional assumptions.

1. It is assumed that normal stresses in the cross sections perpendicular to the shell axis are evenly distributed across the wall thickness (but they are variable about the circumference).

The first assumption can be formulated in another way: the bending moment $\mathrm{M}_{1}$ in the shell wall in the longitudinal direction is assumed to be equal to zero. The normal stresses in the shell cross sections are reduced only to the axial force $N_{l}$, whose intensity is variable about the circumference.

2. Tangential stresses $\tau_{1}$, perpendicular to the median surface and corresponding transverse force $Q_{l}$ are assumed to be equal to zero. Tangential stresses $\tau_{2}$, directed along the circumference are considered to be evenly distributed across the wall thickness.

These stresses are reduced to shift force $S$, whose intensity is also variable about the circumference.

3. The shell is assumed to be non-extensible in the circumferential direction. The relative elongation of the median surface in the circumferential direction is assumed to be equal to zero.

4. The angular deformation of the median surface is also assumed to be equal to zero. This assumption is analogous to the assumption made in the theory of the thin-walled bars bending torsion. According to it, the angular deformation of the median surface is considered to be equal to zero in spite of the presence of the tangential stresses of the bending torsion.

5. The mutual influence of the longitudinal and transverse deformation is not taken into account, i.e. the Poisson coefficient is considered to be equal to zero.

The introduction of all enumerated hypothesis is equivalent to the substitution of the real shell by a design diagram. The shell is represented in it as a totality of a big number of separate nonextensible rings interconnected by joints. These joint connections prevent relative movements in the axial and circumferential directions but do not transmit the radially directed transverse forces and bending moments.

Dependences for the determination of the stresses at shaping were obtained earlier with the use of the assumptions of the technical theory of shells and V.Z. Vlasov's semi-zero-moment theory [10 12]. It was mentioned that when the rollers penetrate into the workpiece, there occur bending moments in the tangential and meridional directions and they introduce certain corrections into the general pattern of stresses distribution. In this case it is necessary to take them into account in the calculation of forces. The determination and division of the locality of the application of the deformation forces are the most important aspects in this method. Let us assume that the surface load is symmetrical in relation to the plane $\varphi=0$. Then the load components $q_{1}, q_{2}, q_{3}$ can be expanded into the following series [13]:

$q_{1}=q_{10}+\sum_{l}^{\infty} \bar{q}_{1 k} \cos k, q_{2}=q_{20}+\sum_{l}^{\infty} \bar{q}_{2 k} \cos k, q_{3}=q_{30}+\sum_{l}^{\infty} \bar{q}_{3 k} \sin k$.

As the distributed load acts in the direction of axis $\mathrm{z}$, its other components $\mathrm{q}_{2}$ and $\mathrm{q}_{3}$ are not taken into account (Fig. 2).

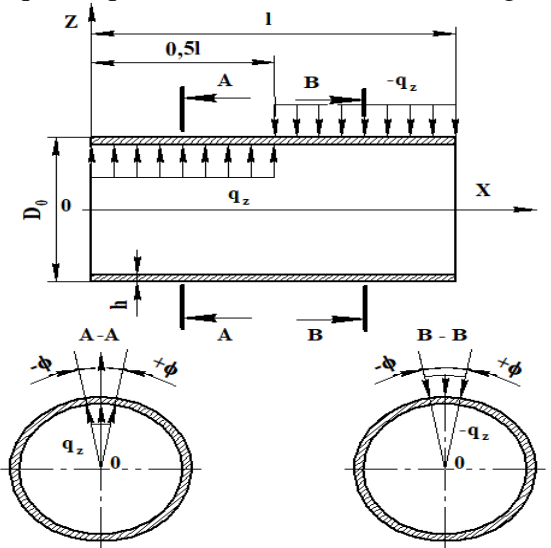

Fig. 2. The scheme of forces action during shaping.
The deforming tool pressure on the cylindrical surface of the workpiece:

at $-\varphi \leq \varphi \leq \varphi_{0}, q=q_{\text {max }}\left(\cos \varphi-\cos \varphi_{0}\right)$,

at $\varphi_{0} \leq \varphi \leq\left(2 \pi-\varphi_{0}\right), q=0$.

We expand the surface load into a series by $\varphi$ :

$q=q_{0}+\sum_{k=1}^{k=\infty} \bar{q}_{k} \cos k$

Integrating the right and the left parts of the equality (7) from 0 to $2 \pi$, we find $q_{0}$ :

$$
\begin{aligned}
& \int_{-}^{+} q_{\max }^{0}\left(\cos \varphi-\cos \varphi_{0}\right) d \varphi=q_{0} 2 \pi, \\
& q_{0}=\frac{q_{\max }}{2 \pi}\left[2 \sin \varphi_{0}-2 \varphi_{0} \cos \varphi_{0}\right] .
\end{aligned}
$$

To determine coefficient $q_{k}$ of the arbitrary term of series we multiply the right and the left parts of equation (7) by $\cos k \varphi$ and integrate from 0 to $2 \pi$ :

$$
\begin{aligned}
& \int_{-}^{+} q_{\max }\left(\cos \varphi-\cos \varphi_{0}\right) \cos k \varphi d \varphi=\int_{0}^{2 \pi} \bar{q}_{k} \cos ^{2} k \varphi d, \\
& \bar{q}_{k}=\frac{2 q_{\max }}{\pi} w_{k} .
\end{aligned}
$$

It is necessary to determine multiplier $w_{k}$, characterizing the boundaries of the plastic deformation zone:

at $\mathrm{k} \neq 1$

$w_{k}=\frac{\sin \left[(k+1) \varphi_{0}\right]}{2(k+1)}+\frac{\sin \left[(k-1) \varphi_{0}\right]}{2(k-1)}-\frac{\sin k \varphi_{0} \cos k \varphi_{0}}{k} ;$

at $\mathrm{k}=1 w_{k}=\frac{\varphi_{0}}{2}-\frac{1}{4} \sin 2 \varphi_{0}$.

We find secant modulus $E_{c}=\frac{\sigma_{i}}{\varepsilon_{i}}$. Relative deformation in the tangential direction at the end of the shaping process is approximately taken as $\varepsilon_{i}$. The final expression for it, taking into account the coefficients of reduction and expansion, will be of the form:

$$
\text { - for the seat zone }
$$

$\varepsilon_{i t}=h_{f}\left(\frac{1}{R_{0}-R_{\text {in }}}-0.57 \frac{\left(R_{0}-R_{\text {in }}\right)}{\left(R_{\text {ou }}+R_{\text {in }}\right)^{2}}\right)$

- $\quad$ for the groove zone

$\varepsilon_{i t}=\frac{\left(R_{0}+R_{o u}\right)\left(R_{o u}+R_{i n}\right)^{2}}{0.57 h_{f}\left(R_{0}-R_{\text {in }}\right)^{2}+\left(R_{0}+R_{\text {in }}\right)\left(R_{o u}+R_{\text {in }}\right)^{2}}-1$.

In the case of radial-rotation shaping, as in the sheet-metal forming operations, different points of the deformation zone receive different deformations and unequal hardening. The yield stress is the function of the coordinates and in the mutual solution of the equations of balance and the equation of plasticity the yield 
stress is to be considered variable. We take into account hardening during shaping with the use of the method described in [14].

The solution to the problem of finding the field of stresses taking into account hardening faces considerable mathematical difficulties. The interconnection of the fields of stresses and the fields of deformations creates most difficulties. The exact solution to the mentioned problem can be obtained for the given particular conditions only by the method of numerical integration, if the deformation process is considered in time [14-16]. However, this method is rather complicated and does not enable obtaining trailing solutions in the form of analytical functions depending on particular conditions of deformation.

For analytical solutions it is necessary to have a formal expression of the hardening curve. The hardening curve in the yield stress deformation intensity coordinates most accurately characterizes the variation of the yield stress in the process of deformation. Here the yield stress depends on all three main deformations. There are solutions for the process of radial-rotation shaping, where the yield stress in the process of deformation is just determined based on the deformations intensity. In this case the dependence is approximated by power functions of the form [17] $\sigma_{i}=A\left(\varepsilon_{i}\right)^{n}, \sigma_{i}=A\left(e_{i}\right)^{n}, \sigma_{i}=A\left(\psi_{i}\right)^{n}$, where $A$ and $n-$ are the constants of the given metal. However, it results in big mathematical difficulties due to the complexity of the interconnection of deformations.

Less accurate though simpler solutions can be obtained with the use of hardening curves in the yield stress - maximal modulo deformation coordinates. In the process of shaping the wheel rim receives the maximal modulo deformations during the expansion and reduction in the tangential direction. These deformations are several times bigger than the meridional and radial deformations. That is why to take into account hardening we use the curves of the first form in the yield stress - relative deformation coordinates with a linear approximation of hardening curve.

For $\sigma_{i}$ we assume an average value of the yield strength by the deformation zone taking into consideration the material hardening by the linear approximation of hardening curve $\sigma_{s}=\sigma_{T 0}+\Pi \varepsilon$ :

- for the seat zone

$\sigma_{s}=\sigma_{T 0}+\frac{\Pi}{2} h_{f}\left(\frac{1}{R_{0}-R_{\text {in }}}-0.57 \frac{\left(R_{0}-R_{\text {in }}\right)}{\left(R_{\text {ou }}+R_{\text {in }}\right)^{2}}\right)$;

- $\quad$ for the groove zone

$$
\begin{aligned}
& \sigma_{s}=\sigma_{T 0}+\frac{\Pi}{2} h_{f} \times \\
& \times\left(\frac{\left(R_{0}+R_{o u}\right)\left(R_{o u}+R_{\text {in }}\right)^{2}}{0.57 s_{i}\left(R_{0}-R_{\text {in }}\right)^{2}+\left(R_{0}+R_{\text {in }}\right)\left(R_{\text {ou }}+R_{\text {in }}\right)^{2}}-1\right),
\end{aligned}
$$

where $\sigma_{\mathrm{T} 0}-$ the extrapolated yield strength; $\Pi-$ the hardening module; $\varepsilon$ - the maximal modulo of deformation.

To find the force of shaping we use Laplace equation for the shell of revolution

$$
\frac{\sigma_{x}}{R_{\rho}}+\frac{\sigma_{y}}{R_{\theta}}=\frac{q}{h},
$$

Then

$$
q_{\max }=\frac{\sigma_{y} h}{R_{\theta}}=\frac{2}{3} \frac{\pi^{2} E_{c} h^{2}}{L^{2}} .
$$

We substitute equation (19) into (7) and have $q=\frac{\pi E_{c} h^{2}}{2 L^{2}}\left[2 \sin \varphi_{0}-2 \varphi_{0} \cos \varphi_{0}\right]+\sum_{k=1}^{k=\infty} \frac{4}{3} \frac{\pi E_{c} h^{2}}{L^{2}} w_{k} \cos k \varphi$,

taking into account the surface of the deformation zone, finally:

$$
\text { - for the seat zone }
$$

$$
\begin{aligned}
& P_{b}=\frac{\pi^{2} E_{c} h^{2} R_{0} \sin \varphi_{0 n}}{2 L_{b}}\left[2 \sin \varphi_{0 n}-2 \varphi_{0 n} \cos \varphi_{0 n}\right]+ \\
& +\sum_{k=1}^{k=\infty} \frac{2}{3} \frac{\pi^{2} E_{c} h^{2} R_{0} \sin \varphi_{0 n}}{L_{b}} w_{k b}
\end{aligned} ;
$$

\section{- for the groove zone}

$$
\begin{aligned}
& P_{g}=\frac{\pi^{2} E_{c} h^{2} R_{0} \sin \varphi_{0 p}}{2 L_{g}}\left[2 \sin \varphi_{O p}-2 \varphi_{0 p} \cos \varphi_{O p}\right]+ \\
& +\sum_{k=1}^{k=\infty} \frac{2}{3} \frac{\pi^{2} E_{c} h^{2} R_{0} \sin \varphi_{O p}}{L_{g}} w_{k g}
\end{aligned}
$$

where $\mathrm{h}$ - the workpiece thickness; $\mathrm{L}_{\mathrm{g}}, \mathrm{L}_{\mathrm{b}}$ - the length of the deformation zone in the zones of the seat and groove, respectively. The total value of the shaping force will be of the form

$$
P=P_{g}+P_{b}
$$

\section{Results and Discussion}

The creation of the calculation methods and software complexes based on them, designed for the application at the stage of the engineering preparation, is a topical problem for the contemporary enterprise. With their use it is possible to choose the equipment, typical accessories, to predict the time of the product manufacturing cycle and the product quality within the shortest period of time [18]. This statement is based on the analysis of the successful operation of foreign enterprises specializing on the production and promotion to the market of vehicle steel wheels (Jantsa (Turkey), Magnetto Wheels (Italy), GKN Wheels (England), Pronor (Poland), Pless (Hungary) and others) in the sphere of quality provision and marketing. These companies have not only completely automated lines for wheel rims production but also applied software complexes providing technical preproduction [19].

The comparison of the theoretical data calculated based on dependence (23) and the experimental data obtained under the production conditions at the lines of wheel rims shaping revealed that they practically coincide. So, for the line producing $4.5 \mathrm{Ex} 16$ rims the divergence is 4 to $8 \%$, and on the line producing DW14x24 rims - up to $11 \%$. The observed certain discrepancy of the results of the experimental and theoretical data can be explained by the adopted assumptions: about the average value of the yield strength by the deformation zone, by the linear approximation of hardening curve, the equality of the relative tangential deformation and the deformation intensity, as well as by the interpretation of the experimental research results. The increase of the deviation for wide-profile wheels must be caused by a more complex shape in the cross section in comparison with narrow-profile rims. It can also be explained by the application of transit shaping machines which differ from console machines in the design of the power shaft drive. That is why in the future it is necessary to improve the calculation method introducing more strict assumptions, boundary conditions and take into account the design features of the equipment. However, the presented research makes it possible to obtain satisfactory results for practitioners and to choose the equipment of the required power. In spite of the seeming complexity the expressions for the calculation of the shaping forces take into account practically all the components of the workflow. They explicitly provide the possibility for the 
control of the process at the design stage changing the values of the components of the formula.

Fig. 3 shows a typical graph of the shaping force for $4.5 \mathrm{Ex} 16$ wheel rim.

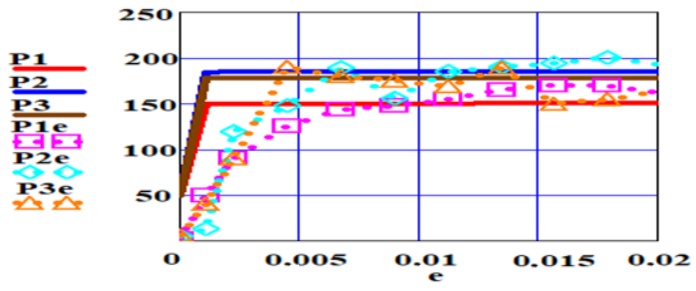

Fig. 3. The calculated and experimental values of the shaping forces at the line of manufacture of 4.5Ex16 rims: $\mathrm{P} 1$ - the calculated value of the force of the first step $(\mathrm{kN}), \mathrm{P} 2$ - the calculated value of the force of the second step $(\mathrm{kN}), \mathrm{P} 3$ - the calculated value of the force of the third step $(\mathrm{kN})$, $\mathrm{P} 1 \mathrm{e}-$ the experimental value of the force of the first step $(\mathrm{kN}), \mathrm{P} 2 \mathrm{e}-$ the experimental value of the force of the second step $(\mathrm{kN}), \mathrm{P} 3 \mathrm{e}-$ the experimental value of the force of the third step $(\mathrm{kN}), \mathrm{e}-$ relative deformation.

It should be noted that we carried out the experimental research not only to verify the theoretical models of the calculation of the shaping forces but also to determine the fields of deformations and stresses during wheel rim shaping under the laboratory conditions $[20,21]$.

\section{Conclusion}

To calculate the shaping force according to the developed method it is necessary: 1. to determine the relative tangential deformations (the analytical dependences obtained as a result of the analysis of the movement of the shape-forming machine power shafts - (1), (2)); 2. to determine the angle of the grip of the workpiece by the rollers - (5), (6); 3. to calculate the multiplier taking into account the locality of the application of the load - (12), (13); 4. to find the secant modulus of the metal based on the indices of the deformations intensity - (14), (15) and the stresses intensity taking into account material hardening - (16); 5. to pass to the calculation of the forces for the expansion and reduction zones - (21), (22); 6 . to determine the total force of deformation - (23).

This method enables the calculation of the required load at the local plastic deformation of the cylindrical workpiece. It bases on the assumptions of the technical theory of shells of revolution and the semi-zero-moment theory of V.Z. Vlasov. One can use it in the design calculations for the technologies of radial-rotation shaping as well as at the creation of applied software for technical preproduction. As known from expressions (21), (22), the force required to give rise to plastic deformation will increase with the growth of the squared thickness and the workpiece radius. It will also decrease with the increase of the length of the deformation zone. The force will grow with every turn as the workpiece secant modulus, characterizing material hardening in the process of deformation, changes.

It provides the possibility to control the value of the required force of deformation at the stage of the workflow design. The specific feature of the developed method consists in taking into account the locality of the load application and the deformation of the workpiece peripheral zones. The method reveals the regularities of the shaping process from the point of view of the closed shell deformation. It is important when taking into consideration all the components of the process as the required force is spent not only on plastic deformation but also on the elastic one that distorts the workpiece as a structure on the whole. This point is taken into account by the second summand in the formulae for the force. Though the relation of the summands in expressions (20), (21) is as 8 to 1 , still the possibility to choose maximum-power machines is significant. It considerably increases the coefficient of the use of the productive capacity and enhances the efficiency of industrial engineering on the whole.

\section{References}

[1] Chigirinsky VV, Legotkin GI, Slepynin AG, Kozlov VI \& Dragobetsky VV (2015), Mechanisms of plastic deformation in case of production of thin-walled rolled stock of the special purpose. Metallurgical and Mining Industry 11, 222 - 230

[2] Wang X, Li L, Deng L, Jin J \& Hu Y (2015), Effect of forming parameters on sheet metal stability during a rotary forming process for rim thickening. Journal of Materials Processing Technology, 223, $262-$ 273, http://doi: 10.1016/j.jmatprotec.2015.04.009

[3] Bi DS, Yang G, Chu L, Zhang J \& Wang ZH (2012), Numerical Simulation on spinning forming process of automotive wheel rim. Materials Science Forum, Switzerland: Trans Tech Publications, 704705 , https://doi.org/10.4028/www.scientific.net/MSF.704-705.1458

[4] Liu Y, \& Qiu X (2016) A theoretical study of the expansion metal tubes. International Journal of Mechanical Sciences, 114, 157-165, https://doi.org/10.1016/j.ijmecsci.2016.05.014

[5] Jurkovic M, Jurkovic Z, Obad M, \& Mustafic E (2015), An investigation of the force and torque at profile sheet metal rolling input data for the production system reengineering. Tehnički vjesnik, 22, 4, 1029-1034, https://doi: 10.17559/TV-20150310092726

[6] Kil T, Lee J, \& Moon Y (2015), Quantitative formability estimation of ring rolling process by using deformation processing map. Journal of Materials Processing Technology, 220, 224-230, https://doi.org/10.1016/j.jmatprotec.2015.01.006.

[7] Bhattacharyya S, Adhikary M, Das MB \& Sarkar S (2008), Failure analysis of cracking in wheel rims - material and manufacturing aspects. Engineering Failure Analysis, 15(5), 547-554, https://doi.org/10.1016/j.engfailanal.2007.04.007

[8] Chigirinskij VV, Mazur VL, Belikov SV, Kolesnik FI, Legotkin GI, Slepynin AG \& Shevchenko TG (2010), Sovremennoe proizvodstvo koles avtotransportny`x sredstv $i$ sel'skoxozyajstvennoj texniki, Dnepropetrovsk: RIA «Dnepr-VAL», pp. 158-204.

[9] Reddy JN (2007), Theory and analysis of elastic plates and shells, CRC Press, Taylor and Francis.

[10] Beben D (2009), Numerical analisis of a soil-steel bridge structure. The Baltic journal of road and bridge engineering, 4, 13-21.

[11] Puzyr` RG, Sosenushkin EN, \& Yanovskaya EA (2013), Ustanovlenie polya napryazhenij pri radial no-rotacionnom profilirovanii cilindricheskoj zagotovki bez ucheta radiusov zakrugleniya deformiruyushhego instrumenta. Vestnik MGTU "Stankin", 4, 42-47.

[12] Shats'kyi IP, \& Makoviichuk MV (2009), Analysis of the limiting state of cylindrical shells with cracks with regard for the contact of crack lips. Strength of Materials, 41, 5, pp. 560-565.

[13] Kalousová K, Souček O \& Čadek O (2012), Deformation of an elastic shell with variable thickness: a comparison of different methods. Geophysical Journal International, 190(2), 726-744, https://doi.org/10.1111/j.1365-246X.2012.05539.x

[14] Abdullah AB \& Samad Z (2007), Cold Forging Die Design: Recent Advanced and Future Trends. Journal of Applied Sciences, 7, 868-876.

[15] Pan SC, Huang MN, Tzou G-Y \& Syu SW (2006), Analysis of asymmetrical cold and hot bond rolling of unbounded clad sheet under constant shear friction. Journal of Materials Processing Technology, 177, 114-120, https://doi.org/10.1016/j.jmatprotec.2006.04.071.

[16] Kukhar V, Artiukh V, Butyrin A, \& Prysiazhnyi A (2018), Stress-Strain State and Plasticity Reserve Depletion on the Lateral Surface of Workpiece at Various Contact Conditions during Upsetting. Advances in Intelligent Systems and Computing, Springer, 692, 201-211, https://doi.org/10.1007/978-3-319-70987-1_22.

[17] Grushko AV, Kukhar VV, \& Slobodyanyuk YO (2017). Phenomenological Model of Low-Carbon Steels Hardening during Multistage Drawing. Solid State Phenomena, 265, 114123, https://doi.org/10.4028/www.scientific.net/SSP.265.114

[18] Kukhar V, Balalayeva E, \& Nesterov O (2017). Calculation Method and Simulation of Work of the Ring Elastic Compensator for Sheet-Forming. $\begin{array}{llll}\text { MATEC Web of Conferences, 129, 01041, } & \end{array}$ https://doi.org/10.1051/matecconf/201712901041.

[19] Wang Z, Zhang Q, Liu Y, \& Zhang Z (2017), Robust and accurate geometric model for automated design of drawbeads in sheet metal $\begin{array}{lll}\text { forming. Computer-Aided Design, } & 92,\end{array}$ http://doi:10.1016/j.cad.2017.07.004

[20] Puzyr RG, Haikova TV, Trotsko OV, \& Argat RG (2016), Determining experimentally the stress-strained state in the radial rotary method of obtaining wheels rims. Eastern-European Journal of Enterprise Technologies, 4, 52-60, http://dx.doi.org/10.15587/17294061.2016.76225.

[21] Puzyr RH, Haikova TV, Majerník J, Karkova M, \& Kmec J (2018), Experimental Study of the Process of Radial Rotation Profiling of Wheel Rims Resulting in Formation and Technological Flattening of the Corrugations. Manufacturing Technology, 18 (1), 106-111. 